\title{
A Química Aplicada ao Estudo das Obras de Arte: O passado E OS DESAFIOS DO PRESENTE
}

\author{
António João Cruz \\ Instituto Politécnico de Tomar; Centro Hércules, Évora \\ ajcruz@ipt.pt
}

\begin{abstract}
Chemistry applied to the study of works of art: the past and the current challenges - The first applications of chemistry to the study of works of art emerged in the late eighteenth century in the context of archaeology. From then on such studies have been performed in a more or less isolated way and only in 1888 the first laboratory has been established in a museum, still with the emphasis on archaeological problems. Considerable changes occurred after the discovery of X-rays, in 1895, with the use of radiography to study paintings, and, after the 1920 s, with the creation of laboratories in museums interested in the conservation of works of art in which research also involved the identification of pigments through micro-chemical tests. Nevertheless, the situation only changed significantly from the 1970s on, first with the appearance of non-invasive equipment and later with the advent of portable equipment that allows non-invasive in situ analysis. The huge technological developments and the important studies already performed, however have not solved certain problems that, in essence, are related to the collaboration between different areas with different cultures.
\end{abstract}

\begin{abstract}
As primeiras aplicações da Química ao estudo das obras de arte surgiram em finais do século XVIII ligadas a contextos arqueológicos. Os estudos foram-se repetindo em grande número, mas de forma mais ou menos isolada, e só em 1888 surgiu o primeiro laboratório num museu, ainda que interessado sobretudo em problemas arqueológicos. Verificaram-se alterações consideráveis depois da descoberta dos raios-X, em 1895, e do uso da radiografia para o estudo de pinturas e, a partir da década de 1920, com a criação de laboratórios em museus especialmente interessados na conservação das obras de arte, nos quais a investigação, além de recorrer a estes métodos, envolvia também a identificação de pigmentos através de testes microquímicos. Contudo, a situação só mudou de forma significativa a partir da década de 1970, em primeiro lugar com o aparecimento de equipamentos não invasivos e, depois, com o aparecimento de equipamentos portáteis que permitiam a análise não invasiva in situ. Os enormes desenvolvimentos tecnológicos e os importantes estudos que estes possibilitaram não resolveram, no entanto, determinados problemas que, no fundo, estão relacionados com a colaboração entre áreas diferentes com culturas diferentes.
\end{abstract}

Os importantes desenvolvimentos tecnológicos dos últimos tempos, e as possibilidades analíticas que abriram, em combinação com algum esquecimento da história, estão na origem da ideia generalizada de que o estudo laboratorial das obras de arte é algo relativamente recente e que a simples análise química dos materiais constituintes das obras de arte é algo de muito importante para a conservação dessas mesmas obras. No entanto, como se pretende dar conta a seguir, esses estudos já têm uma longa história e, por outro lado, a sua relevância para a conservação das obras não é proporcional aos recursos analíticos utilizados, havendo outros aspectos não menos importantes a condicionar o resultado final.

\section{As PRIMEIRAS APLICAÇ̃̃ES DA QUÍMICA AO ESTUDO dO PATRI- MÓNIO ARQUEOLÓGICO}

Foi no último quartel do século XVIII que, no contexto de laboratórios tal como os concebemos hoje, se realizaram as primeiras análises químicas de obras de arte e outros bens culturais. Para isso contribuíram, por um lado, os desenvolvimentos da História da Arte e da Arqueologia que levaram a considerar que a História não se constrói apenas com documentos escritos mas também com as obras [1] e, por outro lado, o grande desenvolvimento que a Química teve nessa ocasião. No entanto, já muitos séculos antes, semelhantes preocupações com a composição das ligas metálicas utilizadas nalgumas obras tinham sido esclarecidas através de procedimentos simples. São exemplos o caso relatado por Vitrúvio, no século I a. C., da determinação por Arquimedes da composição da coroa do rei de Siracusa, a partir da diferente densidade do ouro e da prata [2], e as determinações da pureza de diversos metais, com base nas reacções que apresentam durante o aquecimento, efectuadas segundo as instruções do designado Papiro $X$ de Leyden, do século III [3].

No século XVIII, já no ambiente laboratorial moderno, uma das primeiras análises foi realizada por Alchorn em 1774 (Figura 1) e incidiu sobre duas espadas pré-históricas sobre as quais concluiu:

"It appears to be chiefly copper, interspersed with particles of iron, and perhaps some zinc, but without containing either gold or silver: it seems probable that the metal was cast in its present state, and afterwards reduced to its proper figure by filing. The iron might either have been obtained with the copper from the ore, or added afterwards in the fusion, to give the necessary rigidity of a weapon. But I confess myself unable to determine anything with certainty." [4] 


\section{[ sss ]}

\section{An Account of fome Irith Antiquities. By Governor Pownall.}

\section{Zead at the Socisty of Astieuaries, Febriary 10, t774}

N the courfe of laft year I exhibited to the Society two fwords,
and fome other fragments faid to bave been parts of an
image found in a bog at Cullen, in the county of Tipperary, in
Ireland, on the lands of Lord Milton. Thefe are now in the pof-
feffion of that noble lord; and his politenefs gave me an oppor-
tunity of communicating them.
For the defcriptions of their forms I refer to the drawings [a]
which I then laid before the Society. The fwords were of that
metal which, in our common tranflations of the ancients, we call
brafs. They are not however of that temperament which we
now exprefs by that word. That the Society might have a
precife and philofophic defcription of the metal, I applied to the
maiter of the mint; and by his direction Mr. Alchorn, his Ma-
jefty's aflay-mafter, made an accurate affay of the metal. "It
" appears," he fays, " to be chiefly copper, interfperfed with par-
" ticles of iron, and perbaps fome zinck, but without containing
" either gold or filver: it feems probable, that the metal was caft

[a] See Place XIX.

$$
\mathrm{Z}_{\mathbf{z}} \text { a }
$$

Figura 1 - Primeira página da publicação onde é apresentado o estudo de Alchorn realizado na 2. ${ }^{\text {a }}$ metade do século XVIII [4]

Pouco depois, Johann Friedrich Gmelin analisou mosaicos romanos, identificando, ainda que erradamente, o responsável pela cor azul [1,5]. Em 1795, M. H. Klaproth, que teve um papel de relevo na descoberta de diversos elementos químicos, procedeu à determinação da composição química de algumas moedas gregas e romanas o que, três anos depois [6], originou a primeira de numerosas publicações que dedicou ao estudo analítico de obras da Antiguidade [7]. Por essa ocasião, George Pearson analisou diversos objectos pré-históricos de metal, que, para isso, foi necessário "sacrificar" [8]. A partir de finais do século XVIII, de forma irregular mas sustentada, sobretudo em França, Alemanha e Reino Unido, estudos semelhantes de achados arqueológicos em metal e vidro foram realizados por diversos cientistas $[9,10]$. Não tiveram apenas a intenção de identificar os materiais e as técnicas, mas nalguns casos pretenderam esclarecer questões mais complexas, designadamente de proveniência [11] e de datação.

Em Portugal, estudos análogos parecem terem surgido só em finais do século XIX. Entre esses primeiros trabalhos, contam-se os de Alfredo Bensaúde dedicados à identificação dos materiais usados em diversos objectos pré-históricos, quer através de análise mineralógica (que teve em conta a "impossibilidade de mutilar os objectos preciosos e por vezes únicos”) [12], quer através de análise química propriamente dita (Figura 2) [13], e os de caracterização química de tecidos históricos (fibras, fios metálicos e corantes) por Charles Lepierre [14] - que, pouco depois, estudou a cerâmica portuguesa, não a antiga, mas a da sua época [15].

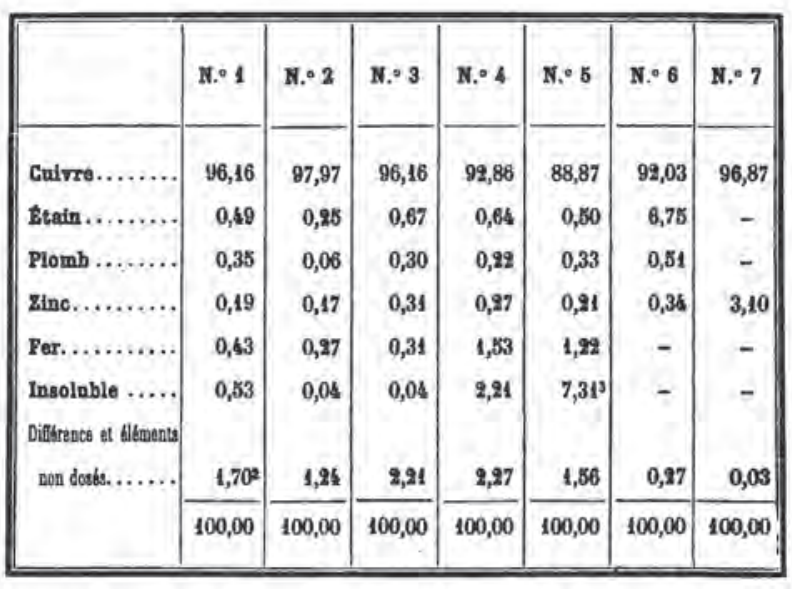

Figura 2 - Resultados das análises químicas efectuadas por Alfredo Bensaúde, em finais do século XIX, a sete objectos pré-históricos de metal encontrados em Portugal [13]

\section{OS PRIMEIROS ESTUDOS ANALÍTICOS DE OBRAS DE ARTE}

O estudo analítico das obras de arte teve início praticamente na mesma ocasião que o estudo dos bens arqueológicos e, nalguns casos, incidiu precisamente sobre obras da Antiguidade reveladas através de trabalhos arqueológicos. O primeiro estudo conhecido em que foi seguida uma abordagem química parece ter sido o de Johann Friedrich Gmelin, em 1781, dedicado à identificação de pigmentos e aglutinante usados na pintura de um sarcófago egípcio [16], ainda que, imediatamente antes, testes simples de solubilidade tenham sido usados com o objectivo de identificar o aglutinante utilizado noutras pinturas [1]. Pretendia-se então mostrar que o uso da pintura a óleo era bem mais antigo do que o registado pelas fontes documentais que atribuíam a sua descoberta a Jan van Eyck, no século XV. O interesse pelo aglutinante de pinturas medievais esteve na origem de diversos estudos que se realizaram até finais do século XVIII, uns com base em análise química, outros com base apenas nos referidos testes de solubilidade efectuados na superfície das obras [1]. No século XIX estes estudos sobre os pigmentos aumentaram em número e profundidade. Entre os que tiveram maior divulgação e impacto contam-se os de Jean-Antoine Chaptal e, sobretudo, os de Humphry Davy $[17,18]$, ambos químicos de renome, sobre os pigmentos utilizados na pintura mural romana, publicados logo no início do século [19,20]. O estudo de Davy é particularmente notável uma vez que, tendo encontrado um pigmento azul desconhecido na sua época, conseguiu identificá-lo como sendo um dos referidos nas fontes documentais da Antiguidade (o azul egípcio).

Até finais do século XIX foram realizados muitos estudos nesta área alguns dos quais por nomes de relevo nas Ciências ou nas Técnicas como, além dos já citados, Louis Nicolas Vauquelin, Michael Faraday ou Michel Eugène Chevreul [1]. No essencial, os métodos de análise usados foram os testes baseados em reacções químicas que se mantiveram em uso até à segunda metade do século XX.

Em Portugal, a primeira identificação dos materiais de uma pintura parece ter sido efectuada em 1923 por Herculano 
de Carvalho, discípulo de Charles Lepierre, por solicitação do conservador Carlos Bonvalot. No entanto, o trabalho ficou inédito várias décadas [21].

De uma forma geral, as investigações orientaram-se para a identificação dos materiais usados nas obras e não para os seus problemas de alteração e de conservação. Contudo, estes também foram abordados durante o século XIX, ainda que tais estudos sejam menos conhecidos por geralmente não terem sido publicados. Um destes estudos foi realizado em meados do século por Michael Faraday com o objectivo de esclarecer a razão de as pinturas da National Gallery, em Londres, se alterarem mais rapidamente do que as de outras colecções, a forma de evitar o problema e o modo de tratar essas obras [22]. Após esse trabalho, afirmou:

I have not the smallest doubt that a person of competent chemical knowledge, and a little acquainted also with the practice of painting in ancient and modern times might be valuably employed in ascertaining such points [the preservation of pictures by means of varnishes or other things]; and I wonder that it should be left, as it were to accident, when accurate knowledge upon the subject might be so easily acquired [22].

Um outro caso relacionado com questões de conservação, este com impacto bem maior, foi o do tratamento com vapores de etanol apresentado em 1870 por Max von Pettenkofer para vernizes com perda de transparência devido à humidade - segundo o mesmo, um problema de "descontinuidade da coesão molecular” [23].

No final do século, o conhecimento já alcançado através da análise química de pinturas antigas, do estudo de problemas de conservação e da produção de materiais para artistas permitiram a A. H. Church publicar, em 1890, um volumoso livro sobre The Chemistry of Paints and Painting [24]. Um dos capítulos é dedicado à apresentação dos materiais e das técnicas de pintura empregues em numerosas obras, ainda que não seja claro o que resultou de análise química ou de outros métodos de investigação. O livro teve grande procura, até 1915 conheceu mais três edições e durante vários anos continuou a ser uma referência sobre o assunto.

\section{OS PRIMEIROS LABORATÓRIOS EM MUSEUS E OS NOVOS MÉTO-} DOS DE EXAME DE OBRAS DE ARTE

Foi também em finais do século XIX que surgiu o primeiro laboratório num museu - o Chemisches Laboratorium Staahche Museum, criado em Berlim em 1888, o qual, durante vários anos, teve como primeiro responsável Friedrich Rathgen [25]. Mais do que o estudo das obras, tinha como objectivo evitar a sua degradação, especialmente os objectos recuperados em escavações arqueológicas no Oriente, como se pode verificar através do manual que publicou sobre os tratamentos efectuados durante os primeiros dez anos de actividade [26,27]. O segundo laboratório, que também teve o mesmo objectivo de conservação, surgiu apenas em
1920, em Londres, no The British Museum, graças ao empenhamento de Alexander Scott, presidente da Chemical Society [28].

Pelo meio, ocorreu a descoberta dos raios-X, em 1895, por Wilhelm Conrad Röntgen, que rapidamente foram usados para a realização de radiografias de pinturas que se mostraram essenciais quer para o diagnóstico do estado de conservação, quer para os estudos relacionados com a autoria e a autenticidade [29]. Depois de um abrandamento destes estudos, os mesmos tornaram-se frequentes após o final da primeira Guerra Mundial, quando o equipamento móvel de radiografia médica começou a ser aproveitado para o exame de obras de arte em vários países da Europa, especialmente em França. A iniciativa alargou-se rapidamente e na década de 1920 surgiram projectos de vulto como o do Fogg Art Museum que tinha como objectivo, entre outros, a constituição de um arquivo de radiografias que pudesse ser útil para a História da Arte [30]. No âmbito deste projecto, Alan Burroughs deslocou-se a alguns dos mais importantes museus europeus, radiografou muitas dezenas de pinturas e, mais tarde, publicou um livro que constitui um marco desta história [31]. Em Portugal, um projecto semelhante foi empreendido, a expensas próprias, pelos médicos Roberto de Carvalho e Pedro Vitorino de 1928 a cerca de 1936, quando foram proibidos de radiografar obras de museus do Estado [29]. Antes, apenas a pintura que tinha sido objecto de análise química por iniciativa de Carlos Bonvalot, em 1923, tinha sido igualmente radiografada [21].

Todos estes trabalhos, realizados um pouco por todo a parte, mostraram a contribuição que a radiografia podia dar ao conhecimento da pintura antiga e, por isso, os museus começaram a adquirir equipamento especializado que foi integrado nos laboratórios criados na mesma ocasião - estes, sim, já especialmente voltados para o estudo das obras de arte. A aquisição do equipamento de radiografia aconteceu no Bayerische Staatsgemäldesammlungen, em Munique, em 1924, no Museu do Louvre e no Fogg Art Museum, em Harvard, em 1926, no Kunsthistorisches Museum, em Viena, em 1930, na National Gallery, em Londres, em 1934, e no Museu Nacional de Arte Antiga, em Lisboa, em 1936, entre outros [29,32].

Enquanto decorreu este processo, ficaram também disponíveis outros métodos de exame que, de uma forma geral, passaram também a equipar tais museus. O primeiro foi a fotografia do trabalho plástico dos pincéis, umas vezes designada como microfotografia (devido à área fotografada), outras como macrofotografia (devido à lente usada), utilizada, pelo menos, desde 1914 por A. P. Laurie como o objectivo de pôr em evidência marcas características de cada artista [33,34], que originou a variante mais geral da fotografia com luz rasante [35]. Outro foi a fotografia de ultravioleta, pela primeira vez aplicada ao estudo de obras de arte por James Rorimer no Metropolitan Museum of Art, em Nova Iorque, em 1929, que mostrou grande utilidade para a distinção de materiais visualmente semelhantes, nomeadamente os usados em restauros [36,37]. Um terceiro método foi a fotografia de infravermelho, usada no estudo 
de pinturas, pelo menos desde 1934, para a visualização de repintes sob vernizes opacos e escurecidos, aproveitando a transparência destes materiais no infravermelho próximo [38]. Depois o método foi usado para observação do desenho subjacente existente nalgumas pinturas [39] e para a identificação de pigmentos [40].

Estes métodos fotográficos e a radiografia, pela informação única que proporcionam, são fundamentais para a compreensão das obras de arte - com a vantagem de serem não invasivos, ou seja, não causarem qualquer dano às obras. Durante várias décadas constituíram o principal recurso analítico de muitos laboratórios associados a museus nalguns casos praticamente o único, como aconteceu no Museu Nacional de Arte Antiga e no Instituto José de Figueiredo, em Lisboa, até cerca de 1970 [41] -, mas, mesmo assim, possibilitaram a obtenção de resultados muito interessantes, inclusivamente para um público geral (Figura 3) $[32,42]$

\section{X-RAYS DETECT ART FAKES BY REVEALING AGE}

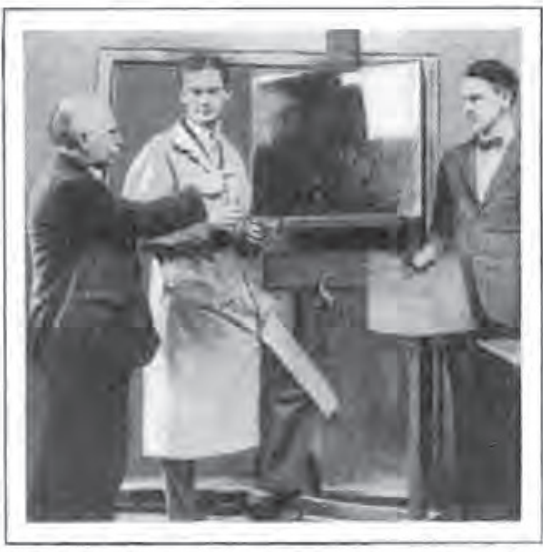

Studying a Painting before Subjecting It to X-Rays, to Test Its Genuineness

Spurious paintings are sometimes detected by $\mathrm{X}$-rays which show a difference in the structure of new and old materials. For instance, an old painting has been retouched by a modern artist to make it resemble the work of a recognized master, or some other method has been followed to disguise a subject. If materials of widely varying age have been employed. the difference is likely to be detected by the penetrating rays and the plotograph that is taken with their aid. If a recent painting is reputed to be old, the fraud can often be discovered by the X-ray comparison with a genuine ancient painting.

Figura 3 - Notícia publicada na revista de divulgação Popular Mechanics, de Março de 1929, que ilustra a difusão que o estudo das obras de arte teve em parte do século XX. Na fotografia, à direita, encontra-se Alan Burroughs

\section{A IDENTIFICAÇÃO SISTEMÁtICA DOS PIGMENTOS E OS ESTUDOS INTEGRADOS}

Simultaneamente com o desenvolvimento e aplicação regular dos métodos de análise citados, no início do século XX a identificação dos pigmentos tornou-se uma actividade mais comum e rigorosa. Para isso muito contribuiu a tese de doutoramento de A. Martin de Wild, publicada em 1929, que pretendeu estabelecer os períodos de utilização de cada pigmento (Figura 4) e, assim, obter uma forma de datação das pinturas [43]. A identificação dos pigmentos foi realizada através de testes conduzidos no microscópio óptico com amostras de dimensão muito pequena recolhidas num grande número de pinturas e a importância dos resultados contribuiu para a adopção do método nos laboratórios dos museus. Até meados da segunda metade do século, este método, dito microquímico, foi o único empregue com tal objectivo em muitos museus. Além dos baixos custos, tem a vantagem de facilmente se poder associar à análise estratigráfica - que é indispensável para o estudo de uma pintura por esta ser constituída por diferentes camadas que, como se vê num corte transversal observado ao microscópio, vão do suporte ao verniz, passando, entre outras, pela camada de preparação e por um número variável de estratos cromáticos com características igualmente diversificadas. A associação entre a análise microquímica e a caracterização da estratigrafia é tão natural que frequentemente se confundem por usarem as mesmas amostras e o mesmo microscópio.

A análise microquímica foi posteriormente aperfeiçoada por Joyce Plesters, da National Gallery, em 1956, que estabeleceu o conjunto de testes que passaram a ser universalmente usados [44] - nalguns laboratórios até há muito pouco tempo. Para os aglutinantes também foram criados testes semelhantes, mas assim caracterizados por Plesters:

The term 'analysis' can hardly be applied to the rather rough and ready tests which are available. The most that can be done is to attempt to classify the medium as glue, egg tempera, oil, or oil/resin mixture [44].

Foi neste contexto de expansão dos métodos de exame e análise que em 1930 se realizou em Roma a Conférence Internationale pour l'Étude des Méthodes Scientifiques Appliquées à l'Examen et à la Conservation des Oeuvres d'Art, organizada pelo então Office International des $\mathrm{Mu}$ sées, em que a colaboração entre cientistas, conservadores e historiadores foi o lema. Dentro deste espírito, surgiu em 1932 a revista Technical Studies in the Field of the Fine Arts, do Fogg Art Museum, de Harvard, publicada até 1942, que muito contribuiu para que a Ciência da Conservação se instituísse como disciplina académica nos Estados Unidos [45].

A acumulação destes estudos analíticos, em paralelo com o estudo das fontes documentais, permitiu a Rutherford J. Gettens e George L. Stout iniciarem nos Technical Studies um conjunto de notas sobre os materiais usados em pintura ao longo da história que, pouco depois, originou um livro (Figura 5) que, durante muitas décadas, constituiu a principal obra de referência sobre o assunto e que, hoje, graças à notável integração de um conjunto muito diversificado de conhecimentos provenientes de diferentes áreas e às sucessivas reedições que tem conhecido, ainda se lê com muito interesse e proveito [46]. (Como curiosidade, pode referir-se que Stout serviu de inspiração para a personagem Frank Stokes do filme The Monuments Men, de George Clooney, interpretada pelo próprio.) 


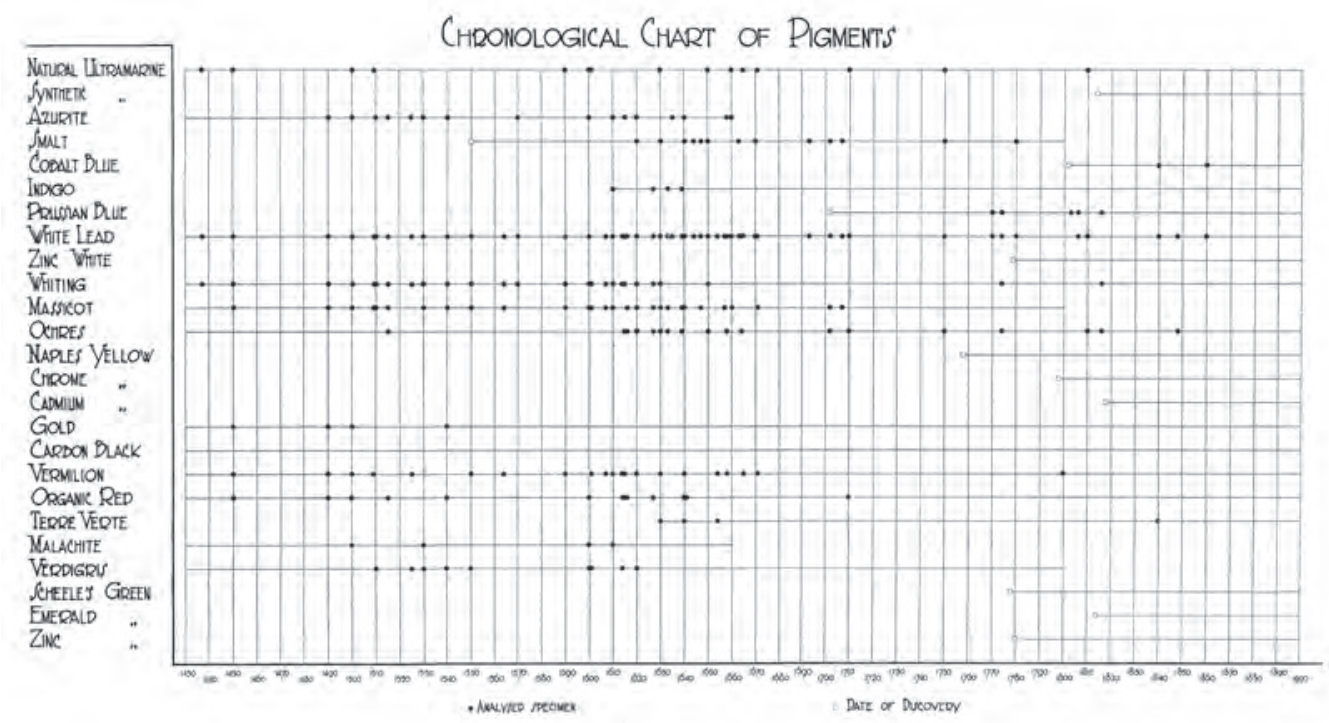

Figura 4 - Esquema com a síntese dos resultados obtidos, em 1929, por A. Martin de Wild relativamente ao período de utilização em pintura dos principais pigmentos [43]

\section{PAINTING MATERIALS A Sbort Encyclopaedia}

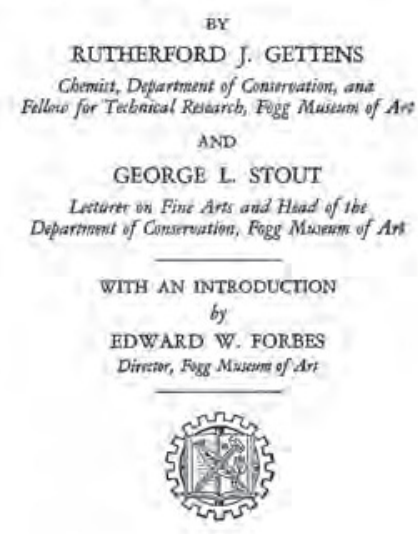

NEW YORK

D. VAN NOSTRAND COMPANY, INC 250 Fouretr AVRUE

Figura 5 - Folha de rosto da 1. ${ }^{\text {a }}$ edição de um livro que, começado a publicar na forma de notas em 1936, ainda hoje constitui uma referência incontornável sobre os materiais usados em pintura ao longo da história [46]

A combinação da análise microquímica e estratigráfica com os referidos métodos de exame permitiu o aparecimento de estudos integrados de pinturas, entre os quais o mais conhecido e que mais contribuiu para o desenvolvimento dos institutos de conservação e restauro e respectivos laboratórios provavelmente foi o efectuado por Paul Coremans, em finais da década de 1940, com o objectivo de esclarecer um importantíssimo caso de falsificação [47]. Esta abordagem foi levada ainda mais longe no estudo de uma pintura do século XV com lugar de relevo na História da Arte ocidental, a Adoração do Cordeiro Místico, de Hubert e Jan Van
Eyck, realizado por um conjunto de pessoas com diferentes formações (Figura 6) [48]. Então, pela primeira vez, uma obra foi estudada segundo os aspectos fundamentais da sua história material, do seu estado de conservação, do seu estilo e da sua técnica e a publicação resultante tornou-se a obra de referência metodológica deste tipo de estudos [49].

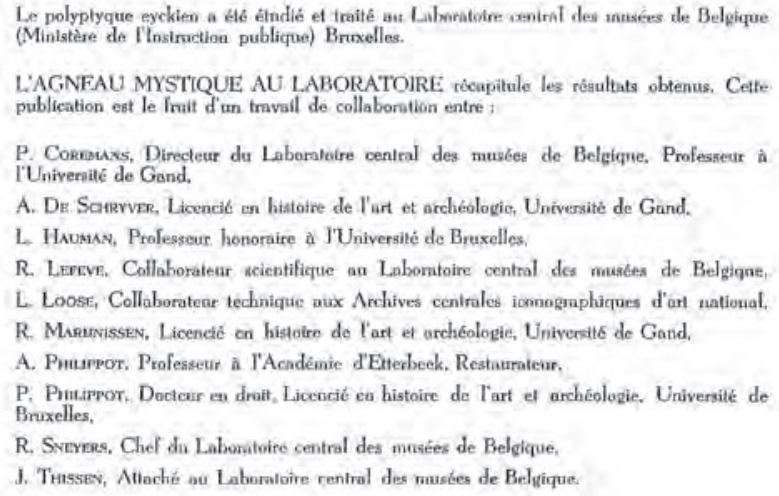

Figura 6 - Ficha técnica sobre a autoria do estudo do políptico da catedral de Gand que instituiu um modelo que continua a ser perseguido [48]

Estes desenvolvimentos vieram a estar na origem da criação do The International Institute for Conservation of Historic and Artistic Works (IIC), em Londres, em 1950, e da sua revista Studies in Conservation, em 1952, que deu continuidade aos objectivos dos Technical Studies.

\section{OS MÉTOdOS ABSOLUTOS DE DATAÇÃO E A ARQUEOMETRIA}

Também a aplicação dos métodos científicos à Arqueologia teve em meados do século XX importantes progressos que igualmente beneficiaram o estudo das obras de arte.

O principal foi o desenvolvimento do método da datação por radiocarbono, em 1949, por W. F. Libby [50] — que por isso recebeu o Prémio Nobel da Química em 1960. Através da análise de materiais orgânicos, o método permitiu, pela 
primeira vez, obter cronologias absolutas e originou uma revolução na Arqueologia [51,52]. As implicações para o estudo das obras de arte, contudo, foram bem mais modestas uma vez que é demasiado grande a incerteza associada a datas recentes.

Pelo contrário, a dendrocronologia, ou seja, o método de datação de madeiras a partir dos seus anéis de crescimento, utilizada para a resolução de problemas arqueológicos no continente americano a partir de 1921 - nessa ocasião através do estabelecimento de cronologias relativas [53] e em 1929 de cronologias absolutas [54] -, teve menos importância nessa área, pelo menos na Europa, devido à inexistência para épocas mais antigas das indispensáveis curvas de referência, mas a partir de 1970 revelou-se como um muito importante método de datação de pinturas sobre suporte de madeira [55]. Além disso, a dendrocronologia permitiu calibrar as datas de radiocarbono, mesmo as mais antigas, a partir do momento em que se descobriu que não se verificava um dos pressupostos necessários para que o método isoladamente pudesse ser empregue de forma absoluta [51].

Um terceiro método de datação que surgiu, este igualmente importante para a datação de cerâmicas arqueológicas e de colecções de artes decorativas, foi a termoluminescência, proposto em 1953 [56] e aplicado a peças históricas em 1968 [57].

Os métodos de datação e a expansão dos métodos espectroscópicos de análise levaram à proliferação das aplicações da Química e da Física à Arqueologia em meados do século XX [58]. Daqui resultou a criação da revista Archaeometry, em 1958, pelo Research Laboratory for Archaeology and the History of Art, de Oxford, que, por sua vez, originou uma nova disciplina - a Arqueometria - que também integra o estudo das obras de arte.

\section{OS MÉTODOS INSTRUMENTAIS DE ANÁLISE E O SEU USO NO ESTUDO DAS OBRAS DE ARTE}

O desenvolvimento geral dos métodos instrumentais de análise que ocorreu ao longo do século XX foi, sem problemas de maior, rapidamente aproveitado pela Arqueologia, mas relativamente às obras de arte a situação foi bem diferente. Por um lado, o acesso a estas é muito mais limitado do que o acesso à generalidade dos bens arqueológicos, não sendo fácil deslocar as obras aos laboratórios onde se encontram os equipamentos analíticos. Por outro lado, a remoção de amostras para análise é no caso das obras de arte extremamente limitada, quer em número, quer em dimensão. Por isso, enquanto os métodos instrumentais eram essencialmente métodos destrutivos ou, não o sendo, requeriam amostras de dimensões relativamente grandes ou envolviam equipamentos não portáteis, o seu uso em obras de arte foi pouco significativo, sobretudo nos casos em que os métodos clássicos eram uma alternativa adequada.

A situação só mudou com o aparecimento de equipamentos que, baseados em métodos não destrutivos, isto é, que não alteram a porção de matéria analisada, permitem fazer a análise directamente sobre a obra, sem remoção de amostra e, assim, sem causar qualquer dano. Ou seja, quando, mais do que usar métodos não destrutivos, foi possível fazer análise não invasiva. Isto aconteceu cerca de 1970 quando surgiu um modelo de espectrómetro de fluorescência de raios-X, pensado para museus, em que o objecto a analisar simplesmente era colocado à frente do equipamento que, por isso, foi designado como "o instrumento de sonho do conservador” [59]. No entanto, devido à natureza elementar do método (que limita os materiais para os quais é adequado) e à reduzida resolução espacial do equipamento, este não substituiu os testes microquímicos na identificação dos pigmentos em pinturas, mas apenas os complementou.

No início da década de 1980 já era relativamente comum o uso de métodos instrumentais de análise no estudo das obras de arte [60]. Porém, devido ao seu elevado custo e à especialização que o seu uso requeria, muito provavelmente na maior parte dos casos esses equipamentos não eram de laboratórios especializados, mas sim de laboratórios de investigação com os quais os museus e os conservadores estabeleceram colaborações. Além disso, estes equipamentos eram usados sobretudo em situações de investigação e não em trabalho de rotina. Obviamente que houve excepções, sendo a mais notória a do Laboratoire de Recherches des Musées de France, em Paris, com o seu acelerador de partículas que ficou operacional em 1988 [32,61].

Só os brutais desenvolvimentos tecnológicos ocorridos recentemente alteraram significativamente a situação, em primeiro lugar, devido à considerável descida de preço dos equipamentos e, em segundo lugar, à miniaturização e à consequente disponibilização de equipamentos portáteis que permitem análises não invasivas in situ (Figura 7). Neste momento, há equipamentos portáteis para grande parte dos métodos de análise relevantes, nalguns casos literalmente equipamentos de mão, ainda que os mesmos, naturalmente, tenham especificações técnicas inferiores às dos equipamentos de bancada. Os métodos actualmente disponíveis e as suas utilizações no estudo das obras de arte são o assunto de um conjunto de manuais que têm surgido nos últimos anos [62-68].

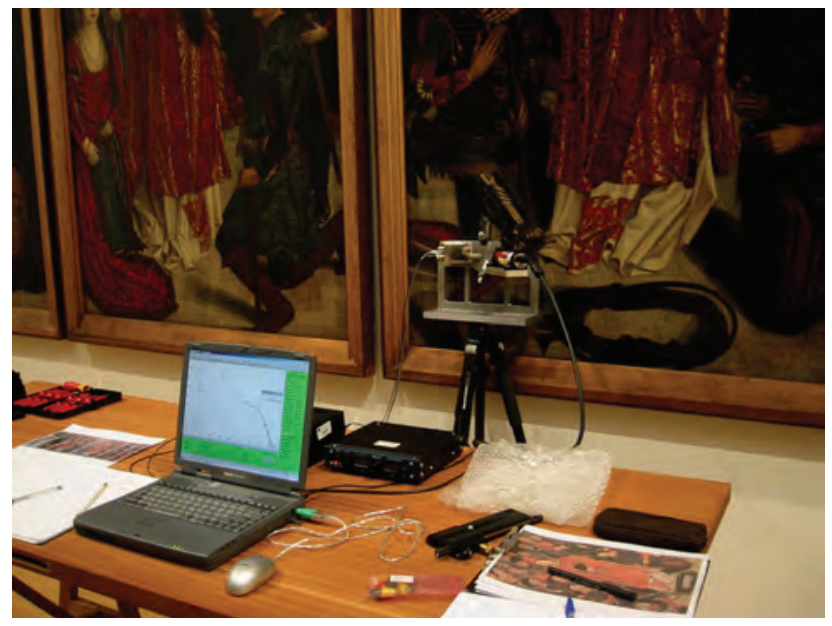

Figura 7 - Análise do Políptico de São Vicente, de Nuno Gonçalves, com equipamento de espectrometria de fluorescência de raios-X portátil 
Por outro lado, as colaborações entre museus e centros de investigação têm-se multiplicado e, assim, as obras de arte têm estado envolvidas em numerosos projectos de investigação - alguns dos quais mudaram completamente o panorama pelo número de pessoas e instituições envolvidas, os recursos empregues e a profundidade dos estudos. O exemplo mais notável é, sem dúvida, o projecto Molart, devido à iniciativa de Jaap J. Boon, que decorreu entre 1995 e 2002 e teve como objectivo abordar ao nível molecular os problemas de conservação das pinturas [69].

\section{Desafios ACtuals}

Não obstante os estudos que têm proporcionado, os desenvolvimentos tecnológicos recentes não só não resolveram alguns problemas antigos da aplicação da Química ao estudo das obras de arte como criaram novos problemas [70]. De uma forma geral, os principais resultam do contexto multidisciplinar: por um lado, as obras tradicionalmente são estudadas e apreciadas no contexto da História da Arte; por outro lado, os métodos analíticos são desenvolvidos e usados no contexto das Ciências.

Um dos problemas que daí deriva tem que ver com as dificuldades de comunicação e colaboração que resultam de as duas áreas terem culturas muito diferentes, por vezes quase opostas [71]. Essa diferente forma de ver o mundo, a que se junta o geral desconhecimento que uma área tem da outra, leva a que ocorram dificuldades de comunicação que, por vezes, originam situações delicadas e põem em causa a colaboração e os próprios estudos. Os conservadores-restauradores estão melhor posicionados, entre as duas áreas, para liderar esse diálogo, mas, conforme o perfil da sua formação, mais marcado pelas Ciências ou mais marcado pelas Humanidades, acabam também por se confrontarem com a outra área. Sobre este problema são claras as palavras de J. R. J. van Asperen de Boer - que na década de 1960 inventou a reflectografia de infravermelho, um avanço relativamente à fotografia de infravermelho -, que em 1998 fazia assim um balanço sobre o impacto das Ciências na História da Arte:

The "Oxford Dictionary" defines 'impact' as 'striking (on, against), collision, effect, influence'. The author has held a chair of 'scientific examination of works of art', being a physicist by training but operated professionally for more than twenty years within an institute for Art History, and would obviously prefer 'effect' or at least 'influence' as the accepted connotation. Unfortunately endeavours to use - or even better - integrate methods of scientific examination in art history are not infrequently seen as 'striking against' art historical views or traditions and may thus well lead to 'collisions' [72].

Os novos desenvolvimentos tecnológicos só aumentaram essas dificuldades de comunicação, pois, como o mesmo van Asperen de Boer referiu no balanço do já citado projecto Molart,

the specialized language used by the participating scientists is not easily grasped by restorers and art histori- ans, not even by the present writer trained as an experimental physicist [73].

Outro problema resulta da aplicação da Química ao estudo das obras de arte por vezes se inserir em projectos desenvolvidos na área das Ciências que, além de pretenderem alargar as possibilidades de financiamento através de outras áreas, pretendem mostrar as possibilidades dos equipamentos analíticos. Ou seja, o estudo das obras não parece ser um fim, mas sim um meio. Além disso, por regra estes estudos originam apenas publicações em revistas de Química, Física ou Materiais que colocam a tónica na novidade da abordagem ou dos equipamentos e deixam pouco espaço para o estudo detalhado das obras. A este respeito é elucidativa a profusão de artigos com títulos que enumeram os métodos analíticos empregues, mas não identificam a obra estudada ou os seus problemas. Acresce ainda que muitas das pinturas que interessa estudar, seja do ponto de vista da História da Arte ou da Conservação, colocam problemas que podem ser respondidos com análises de rotina que dificilmente originam artigos susceptíveis de serem aceites nas revistas que são valorizadas no momento da avaliação de um projecto. Aliás, muita da informação analítica sobre obras de arte, com interesse significativo para o conhecimento destas, não tem sido publicada, também devido ao reduzido hábito de publicação dos conservadores, e faz parte da enorme massa da literatura cinzenta da Conservação e Restauro. Em contrapartida, há muitos artigos em excelentes revistas sem qualquer impacto directo nas obras a que se dedicam.

Os principais desafios que actualmente se colocam à aplicação da Química ao estudo das obras de arte passam, portanto, pela verdadeira conciliação dos diferentes conhecimentos, competências e interesses.

\section{REFERÊNCIAS}

[1] J. Nadolny, Rev. Conserv. 4 (2003) 39-51

[2] Vitrúvio, “Tratado de Arquitectura”, IST Press, Lisboa, 2006, 327

[3] R. Carvalho, “A Ciência Hermética”, Relógio de Água, Lisboa, 1996

[4] T. Pownall, Archaeologia 3 (1775) 355-370

[5] J.F. Gmelin, Commentationes Societatis Regiae Scientiarum Gottingensis 2 (1779) 41-64

[6] M. Klaproth, Mémoires de l'Académie Royale des Sciences et Belles-Lettres 45 (1798) 97-113

[7] E.R. Caley, J. Chem. Educ. 26 (1949) 242-268

[8] G. Pearson, Philos. T. R. Soc. Lond. 86 (1796) 396-451

[9] E.R. Caley, J. Chem. Educ. 28 (1951) 64-66

[10] E.R. Caley, J. Chem. Educ. 44 (1967) 120-123

[11] E. Pernicka, Provenance Determination of Archaeological Metal Objects, in B.W. Roberts, C.P. Thornton (ed.), “Archaeometallurgy in Global Perspective”, Springer, New York, 2014, 239-268

[12] A. Ben-Saude, Note sur la nature minéralogique de quelques instruments de pierre trouvés en Portugal, in 
«Congrès International d'Anthropologie et d'Archéologie Préhistoriques», Lisbonne, 1884, 682-697

[13] A. Ben-Saude, Communicações da Comissão dos Trabalhos Geológicos de Portugal 2 (1892) 119-124

[14] C. Lepierre, O Instituto 42 (1895) 17-22

[15] C. Lepierre, "Estudo Chimico e Technologico sobre a Ceramica Portugueza Moderna”, Imprensa Nacional, Lisboa, 1899

[16] J.F. Gmelin, Commentationes Societatis Regiae Scientiarum Gottingensis 4 (1781) 3-25

[17] S.G. Rees-Jones, Stud. Conserv. 35 (1990) 93-101

[18] M.H.R. Beltran, Quím. Nova 31 (2008) 181-186

[19] J.-A. Chaptal, Ann. Chim. 70 (1809) 22-31

[20] H. Davy, Philos. T. R. Soc. Lond. 105 (1815) 97-104

[21] C. Bonvalot, Arquivo de Cascais 3 (1982) 19-42

[22] N.S. Brommelle, Stud. Conserv. 2 (1956) 176-188

[23] M. Pettenkofer, «Über Ölfarbe und Conservirung der Gemälde-Gallerien Durch das Regenerations-Verfahren», Braunschweig, 1870

[24] A.H. Church, "The Chemistry of Paints and Painting”, Seeley and Co., London, 1890

[25] M. Gilberg, J. Am. Inst. Conserv. 26 (1987) 105-120

[26] F. Rathgen, «Die Konservirung von Alterthumsfunden», Berlin, 1898

[27] F. Rathgen, “The Preservation of Antiquities”, Cambridge University Press, 1905

[28] A.E. Werner, R.M. Organ, Stud. Conserv. 7 (1962) 75-87

[29] A.J. Cruz, Conservar Património 11 (2010) 13-32

[30] F.G. Bewer, “A Laboratory for Art. Harvard’s Fogg Museum and the Emergence of Conservation in America, 1900-1950”, Harvard Art Museum, Cambridge, 2010

[31] A. Burroughs, “Art Criticism from a Laboratory”, Little, Brown and Co., Boston, 1938

[32] J.-P. Mohen, «Les Sciences du Patrimoine», Éditions Odile Jacob, Paris, 1999

[33] A.P. Laurie, "The Pigments and Mediums of the Old Masters”, Macmillan, London, 1914

[34] A.P. Laurie, Nature 92 (1914) 558-560

[35] J.F. Cellerier, Mouseion 13-14 (1931) 3-20

[36] J.J. Rorimer, The Metropolitan Museum of Art Bulletin 24 (1929) 185-186

[37] J.J. Rorimer, "Ultra-violet Rays and their Use in the Examination of Works of Art”, The Metropolitan Museum of Art, New York, 1931

[38] R.A. Lyon, Technical Studies in the Field of the Fine Arts 2 (1934) 203-212

[39] S. Keck, Technical Studies in the Field of the Fine Arts 9 (1941) 145

[40] W. Clark, "Photography by Infrared”, John Wiley \& Sons, New York, 1946
[41] A.J. Cruz, Do certo ao incerto: o estudo laboratorial e os materiais do políptico de S. Vicente, in J.A.S. Carvalho, I. Cordeiro (ed.), "Nuno Gonçalves. Novos Documentos", IPM, Lisboa, 1994, 41-45

[42] M. Hours, "Les Secrets des Chefs-d'Oeuvre”, Robert Laffont, Paris, 1964

[43] A.M. Wild, "The Scientific Examination of Pictures”, G. Bell \& Sons, Ltd., London, 1929

[44] J. Plesters, Stud. Conserv. 2 (1956) 110-157

[45] M.A. Ainsworth, Conservation - The Getty Conservation Institute Newsletter 20 (2005) 4-10

[46] R.J. Gettens, G.L. Stout, “Painting Materials”, D. Van Nostrand Company, New York, 1942

[47] P.B. Coremans, "van Meegeren’s Faked Vermeers and de Hooghs. A scientific examination”, J. M. Meulenhoff, Amsterdam, 1949

[48] P. Coremans (ed.), "L’Agneau Mystique au Laboratoire”, De Sikkel, Anvers, 1953

[49] C. Périer d’Ieteren, "La Restauration en Belgique de 1830 à nos Jours”, Mardaga, Liège, 1991

[50] J.R. Arnold, W.F. Libby, Science 110 (1949) 678-680

[51] J.M.P. Cabral, Química e Pré-história: datação pelo radiocarbono, in A.R. Dias, J.J.M. Ramos (ed.), “Química e Sociedade”, SPQ - Escolar Editora, Lisboa, 1990, 89-144

[52] J.M.P. Cabral, “A Radioactividade. Contributos para a História da Arte”, IST Press, Lisboa, 2011

[53] A.E. Douglass, Natural History 21 (1921) 27-30

[54] A.E. Douglass, National Geographic Magazine 56 (1929) 736-770

[55] J. Bauch, D. Eckstein, Stud. Conserv. 15 (1970) 45-50

[56] F. Daniels, C.A. Boyd, D.F. Saunders, Science 117 (1953) 343-349

[57] M.J. Aitken, D.W. Zimmerman, S.J. Fleming, Nature 219 (1968) 442-445

[58] A.M. Pollard, C. Heron, “Archaeological Chemistry”, RSC Publishing, Cambridge, 2008

[59] V.F. Hanson, The curator's dream instrument, in W.J. Young (ed.), "Applications of Science in Examination of Works of Art”, Museum of Fine Arts, Boston, 1973, 18-30

[60] N.S. Baer, M.J. Low, Advances in scientific instrumentation for conservation: an overview, in N.S. Brommelle, G. Thomson (ed.), "Science and Technology in the Service of Conservation”, IIC, London, 1982, 1-4

[61] M. Menu, Nucl. Instrum. Meth. B 45 (1990) 597-603

[62] J. Janssens, R. Van Grieken (ed.), "Non-destructive Microanalysis of Cultural Heritage Materials”, Elsevier, Amsterdam, 2004

[63] B. Stuart, "Analytical Techniques in Materials Conservation”, John Wiley \& Sons, Chichester, 2007

[64] P. Craddock, "Scientific Investigation of Copies, Fakes and Forgeries”, Butterworth-Heinemann, Oxford, 2009

[65] D. Pinna, M. Galeotti, R. Mazzeo (ed.), "Scientific Exami- 
nation for the Investigation of Paintings”, Centro Di, Firenze, 2009

[66] G. Artioli, "Scientific Methods and Cultural Heritage”, Oxford University Press, Oxford, 2010

[67] H. Edwards, P. Vandenabeele (ed.), “Analytical Archaeometry. Selected Topics”, RSC Publishing, Cambridge, 2012

[68] E.A. Varella (ed.), “Conservation Science for the Cultural Heritage”, Springer, Heidelberg, 2013

[69] M. Clarke, J. Boon (ed.), "MOLART. A multidisciplinary NWO PRIORITEIT project on Molecular Aspects of Ageing in Painted Works of Art. Final Report and Highlights. 1995-2002”, FOM Institute, Amsterdam, 2003

[70] A.J. Cruz, Ciência e Conservação: alguns problemas de uma relação frequentemente conflituosa, mas necessária, in G.V.e. Sousa, E. Vieira (ed.), "Actas do I Encontro Luso-Brasileiro de Conservação e Restauro”, CITAR, Porto, 2012, 15-24

[71] C.P. Snow, "The Two Cultures and the Scientific Revolution”, Cambridge University Press, New York, 1961

[72] J.R.J.A. Boer, Some reflections upon the impact of scientific examination on art historical research, in E. Hermens (ed.), “Looking Through Paintings”, de Prom PublicationsArchetype Publications, Baarn-London, 1998, 13-17

[73] J.R.J.A. Boer, Reflections on MOLART, in M. Clarke, J. Boon (ed.), "MOLART. A multidisciplinary NWO PRIORITEIT project on Molecular Aspects of Ageing in Painted Works of Art. Final Report and Highlights. 1995-2002”, FOM Institute, Amsterdam, 2003, 9-10

\section{Actualidades CientíFicas}

\section{LIBERTAÇ̃̃O DE FRAGÂNCIAS A PARTIR DE MATRIZES DE LÍQUIDOS IÓNICOS}

Químicos no Reino Unido desenvolveram um novo sistema de libertação de fragrâncias que permite manter o aroma no ar durante mais tempo.

Os compostos das fragâncias, naturais e sintéticas, possuem uma multiplicidade de grupos funcionais, tais como álcoois, aldeídos, cetonas, ésteres, lactonas, éteres e nitrilos. As fragrâncias da família dos álcoois são tipicamente bastante voláteis, e o seu aroma desaparece rapidamente após a aplicação do perfume. Muitas pesquisas têm sido dedicadas a encontrar formas de manter o aroma perfumado durante muito mais tempo, tipicamente através da encapsulação dos compostos activos em matrizes adequadas.

Num estudo recentemente publicado, liderado por Nimal Gunaratne da Queen's University Ionic Liquid Laboratories (QUILL) em Belfast, Irlanda do Norte, foi desenvolvida uma via alternativa que passa pela ligação química entre a fragrância (no estudo foram usados dois álcoois, um deles o 2-feniletanol, que tem aroma a rosas) a uma matriz de líquidos iónicos com formação de um hemicetal. Nesta forma, o sistema pode ser visto como uma pró-fragrância, sem aroma. No contacto com a água, o hemicetal é hidrolisado e a fragrância é libertada.

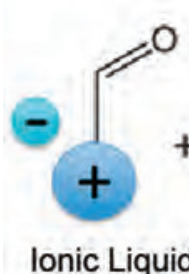

Ionic Liquid

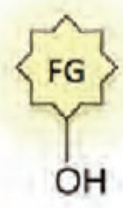

$\mathrm{OH}$

Hemiacetal

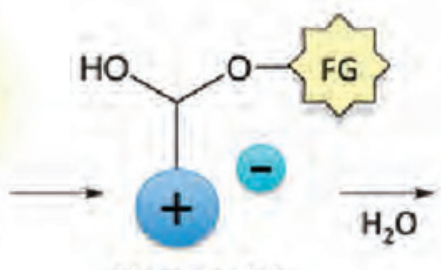

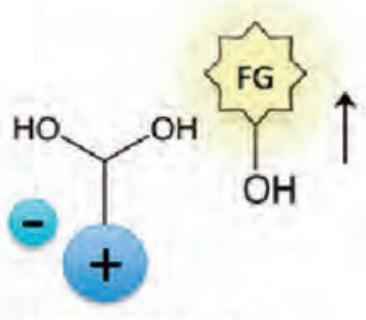

Fragrance release

Os estudos cinéticos realizados mostraram que a fragrância é libertada quantitativamente e que a velocidade de libertação é dependente da quantidade de água presente. Uma aplicação potencial deste sistema é nos desodorizantes, onde o suor poderá fornecer a água necessária para a reacção. O grupo também descobriu que tióis (componentes do suor responsáveis pelo seu odor) também podem desencadear a libertação da fragrância. Isto significa que este sistema pode funcionar como uma espécie de 'dois em um’ contra os odores corporais, através da remoção simultânea do aroma desagradável do suor e libertação dos aromas agradáveis da fragrância.

(adaptado de "Ionic liquids come up smelling of roses", http://www.rsc.org/chemistryworld/2015/04/ionic-liquid-water-trigger-perfume; H.Q. N. Gunaratne, P. Nockemann, K.R. Seddon. Chem. Commun., 51 (2015) 4455-4457 - DOI: 10.1039/c5cc00099h)

Paulo Mendes (pjgm@uevora.pt) 

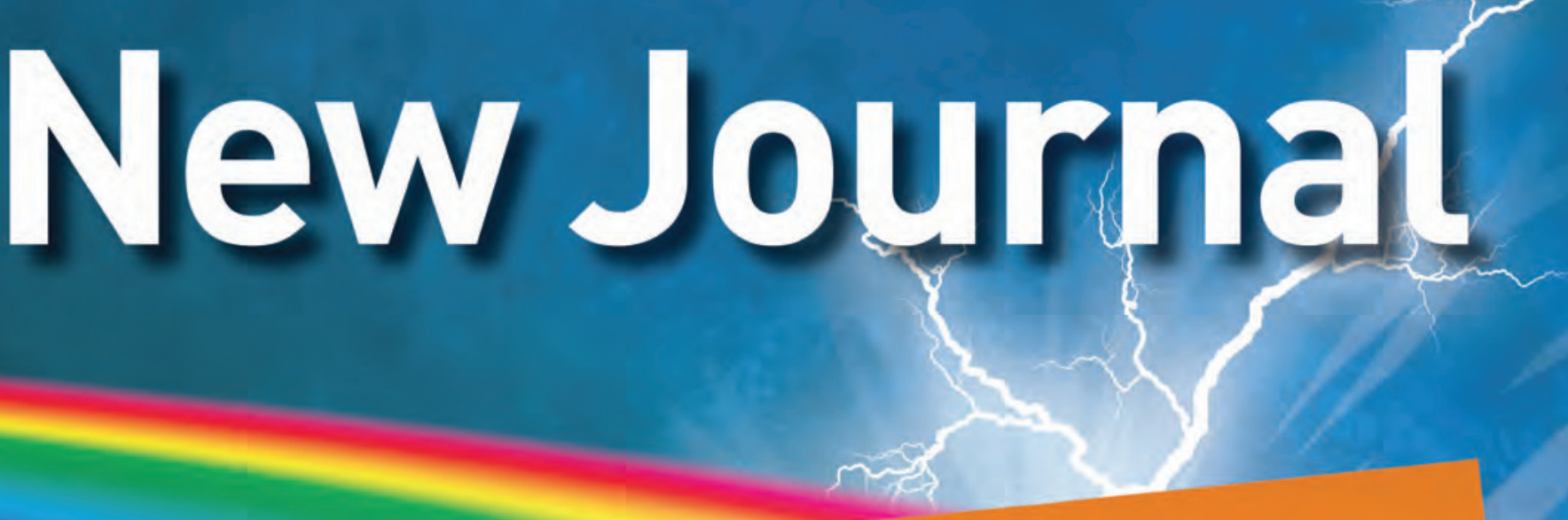

\section{ChemElectroChem}

is a sister journal of:
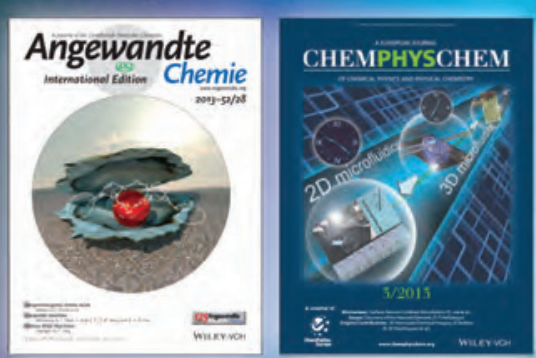

Co-Chairs of the Editorial Advisory Board:

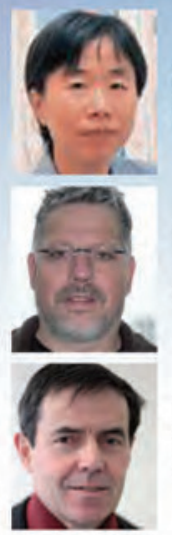

Bing-Wei Mao

(Xiamen University, China)

Wolfgang Schuhmann

(Ruhr-Universität Bochum, Germany)

Jean-Marie Tarascon

(Université de Picardie, France)

CHEMELECTROCHEM

ANALYSIS \& CATALYSIS. BIO \& NANO, ENERGY \& MORE

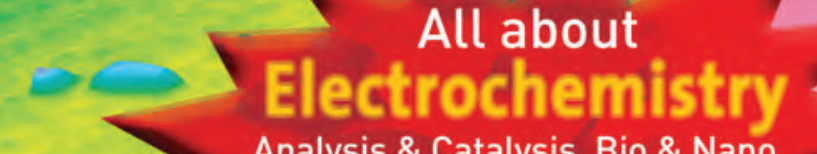
Analysis \& Catalysis, Bio \& Nano,

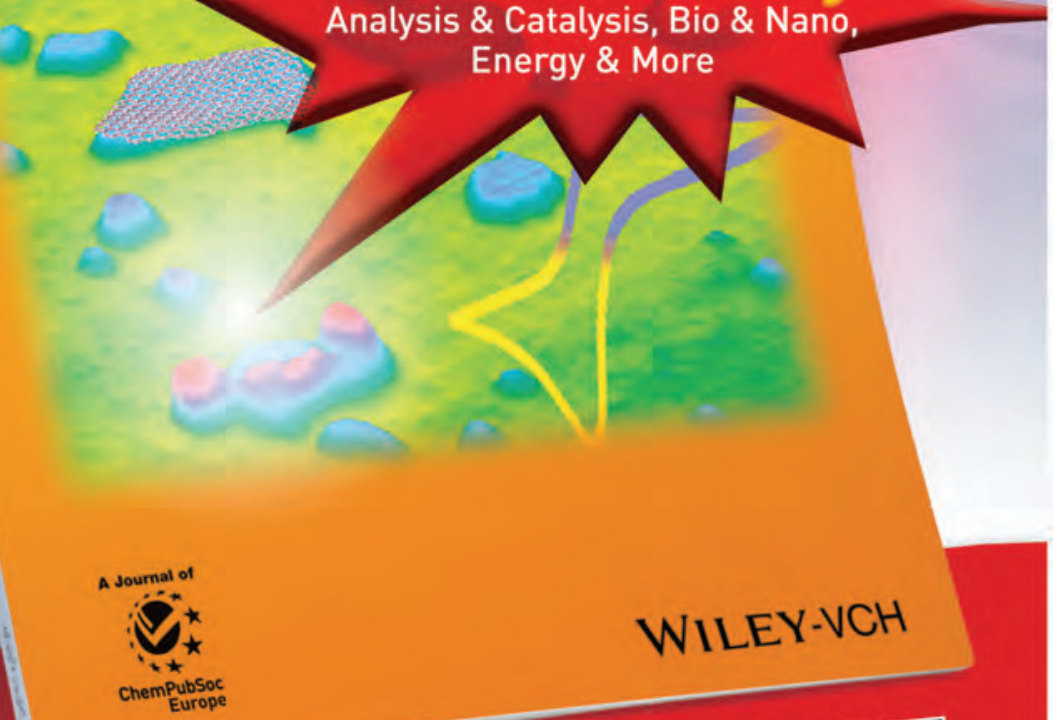

Free Online Access

In 2014 for all users from institutions that have registered Ask your librarian to register for complimentary online access TODAY www.wileyonlinelibrary.com/newjournals-optin

\section{ChemElectroChem}

is a journal of ChemPubSoc European organization comprising 16 European chemical societies.

www.chempubsoc.eu

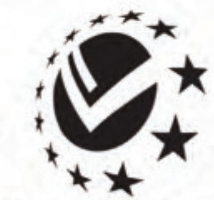

ChemPubSoc

Europe

\section{www.chemelectrochem.org}

WILEY-VCH 